\title{
Vulval aggressive angiomyxoma - a rare entity
}

\begin{abstract}
Aggressive Angiomyxoma is a very rare, distinctive, locally invasive soft tissue tumour, that occurs most commonly in the perineum, pelvis, vulva and vagina. It is seen mostly in women of reproductive age group. It has a tendency for recurrence locally, treatment being surgical excision. We hereby describe a case of 38 -year-old patient with a pedunculated growth on the right labium majora.
\end{abstract}

Keywords: angiomyxoma, pedunculated, vulva
Volume II Issue 4 - 202I

\author{
Jayakar Thomas \\ Emeritus Professor, The Tamil Nadu Dr. MGR Medical University, \\ India
}

Correspondence: Jayakar Thomas, Emeritus Professor, The Tamil Nadu Dr. MGR Medical University, India,

Email jayakarthomas@gmail.com

\section{Introduction}

Aggressive Angiomyxoma is an uncommon, slowly growing myxoid, mesenchymal neoplasm. It is known to most frequently occur in the pelvic, perineal and vulvovaginal regions. It has a high recurrence but a very low tendency to metastasize. It was first reported by Steeper and Rosai in 1983. ${ }^{1}$ It usually presents as a polyp or pedunculated growth of the vulva and is diagnosed on Histopathological examination. Estrogen and progesterone receptors are found commonly in Angiomyxomas. ${ }^{2}$ It is therefore most likely to grow during pregnancy and during hormonal therapy. Surgical excision is most preferred treatment whereas other modalities may also be needed.

\section{Case report}

A 38 year old female patient presented to our Skin OPD with a swelling in the genitalia in the last 3 years. It was slowly growing throughout and increased in size from the last 6 months. There was no history of pain, discharge from lesion, vulval discharge, bleeding, ulceration, sexual difficulty, constitutional symptoms, except for a feeling of something hanging while standing and walking. She had regular menstrual cycles.

Local examination showed a well circumscribed pedunculated polypoidal fleshy mass measuring $4 \times 5 \mathrm{~cm}$, with a thickened stalk of $8 \mathrm{~cm}$. On palpation, it was non tender with a soft and spongy consistency. The tumour was lobulated and had few well-defined depigmented patches and macules. Inguinal lymph nodes were not enlarged. Vagina and rest of vulva was normal.

The tumour was surgically removed and sent for histopathological examination. On Histopathology, epidermal atrophy was seen. Dermis showed numerous thick-walled blood vessels in the background of myxoid stroma admixed with stellate and spindle shaped fibroblasts. Based on these features, we came to a diagnosis of Aggressive Angiomyxoma.

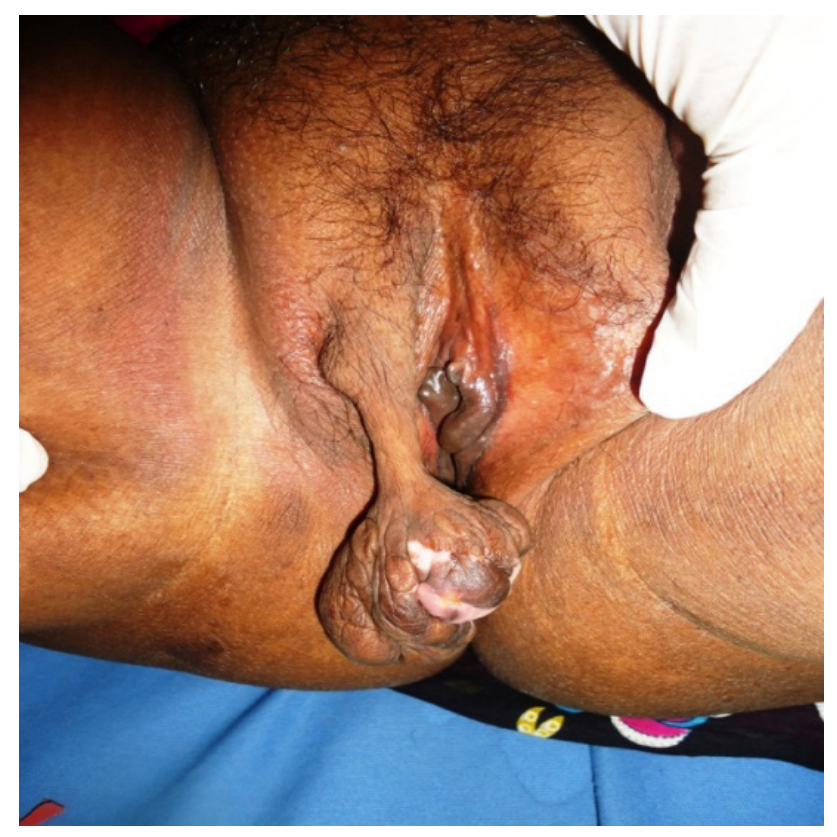

Figure I A well-defined pedunculated growth measuring $4 \times 5 \mathrm{~cm}$, with the stalk measuring $8 \mathrm{~cm}$ seen arising from right labium majora. 


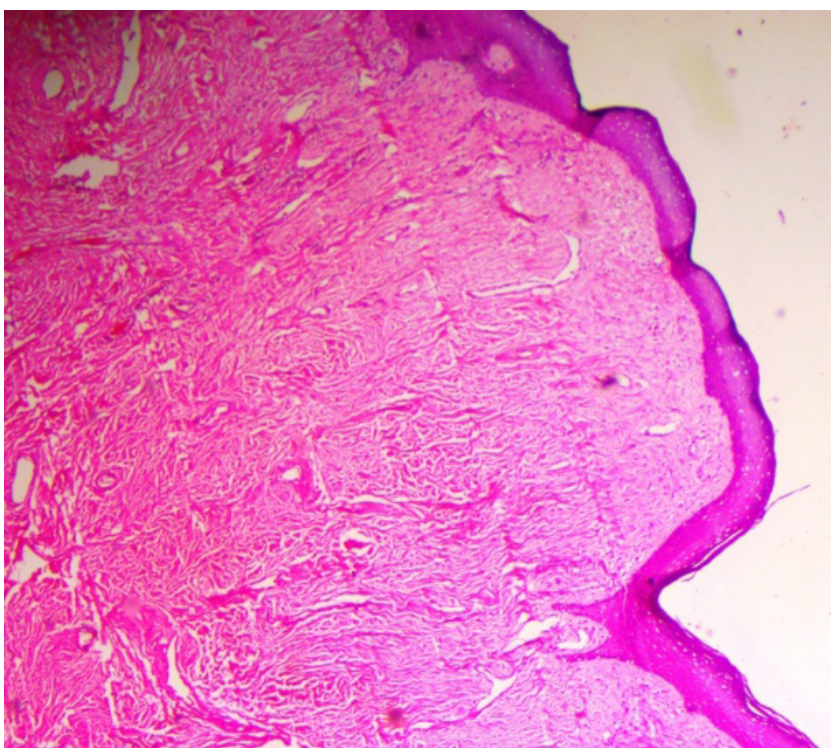

Figure 2 Histopathology in Scanner view shows epidermal atrophy, multiple blood vessels in myxoid stroma, H\&E staining.

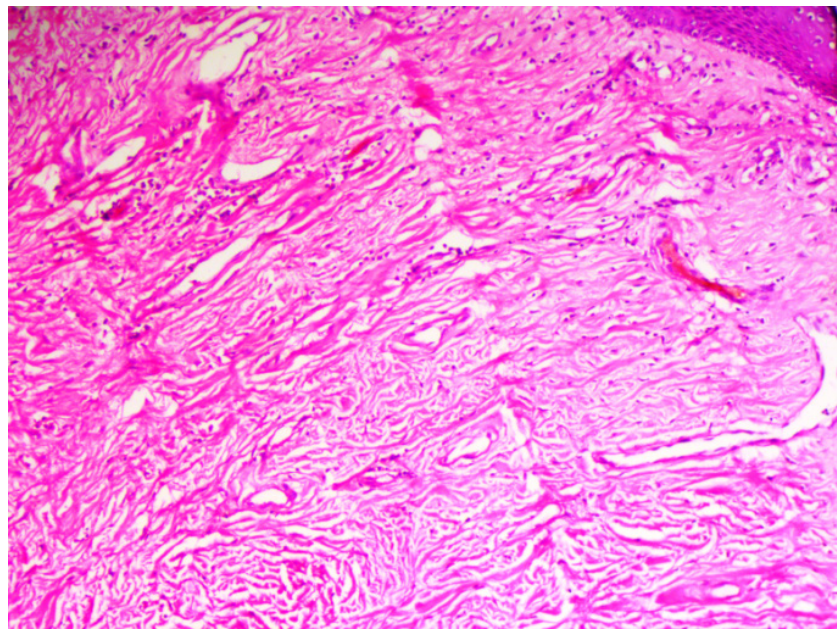

Figure 3 Low power view showing numerous thick-walled blood vessels in the dermis, H\&E staining.

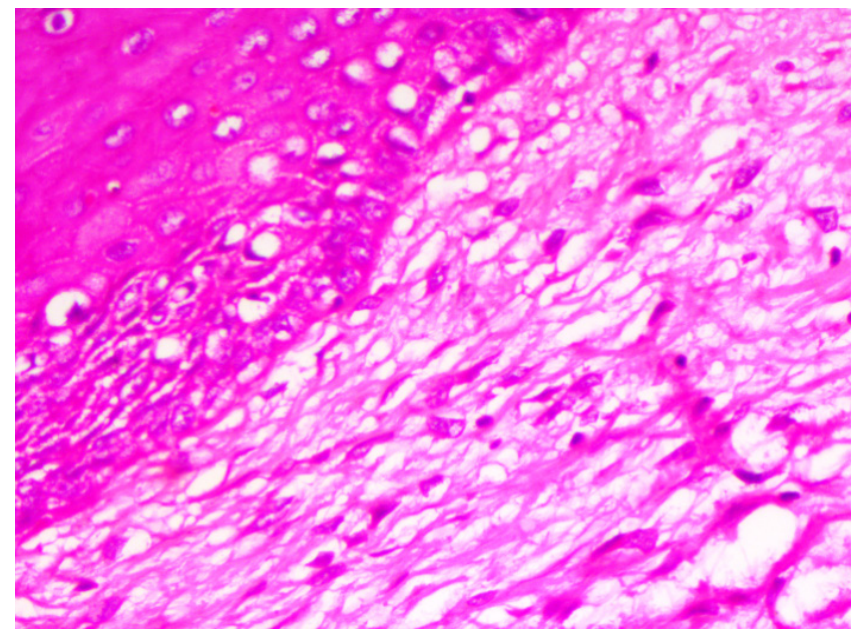

Figure 4 Higher magnification showing stellate and spindle shaped fibroblasts, H\&E staining.

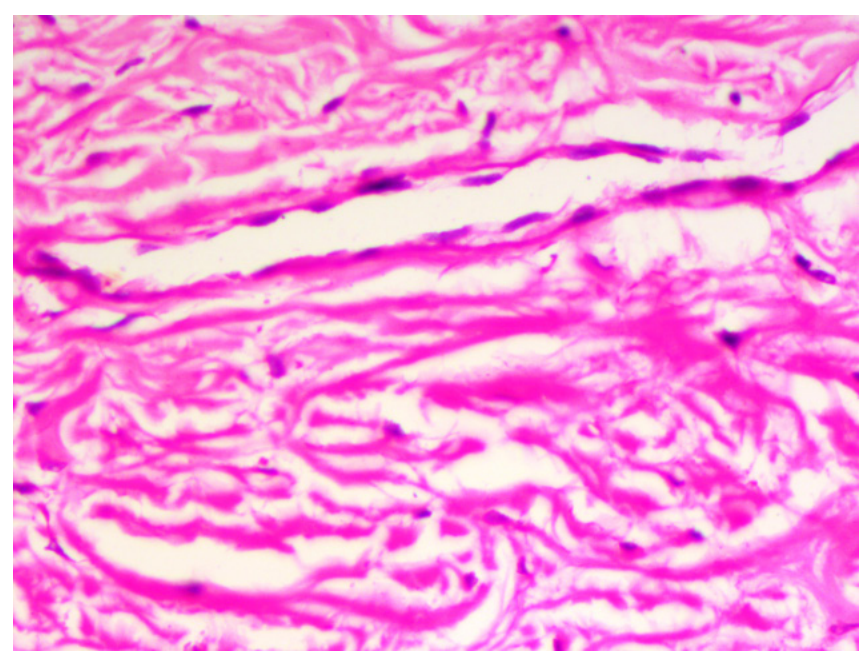

Figure 5 Histopathology showing thick lining of the blood vessels, H\&E staining.

\section{Discussion}

Aggressive Angiomyxoma presents as an asymptomatic mass in the genital region of women in reproductive age group, occasionally reported in males (M:F=1:6). ${ }^{2}$ It is usually termed as "aggressive" which denotes its tendency to be locally invasive and for its recurrence after removal. Common sites of occurrence include vulva, vagina, perineum, pelvis and urinary bladder.

Angiomyxomas are classified as Superficial or Cutaneous myxomas and Aggressive Angiomyxomas. Superficial angiomyxomas present in middle aged adults as a single polypoidal lesion in the head and neck region, and maybe confused with Acrochordon or Neurofibroma, on the other hand Aggressive Angiomyxomas are seen mostly in women of reproductive age, distributed in the pelvic-perineal regions. ${ }^{2}$

Aggressive Angiomyxomas are hormonally responsive neoplasms believed to be arising from specialized mesenchymal cells or multipotent perivascular progenitor cells in the pelvic-perineal region which have variable myo-fibroblastic features or can be even due to translocation of chromosomal $12{ }^{3}$

Aggressive Angiomyxoma in the vulva maybe misdiagnosed as Lipofibroma, Bartholin cyst and Labial cyst. Angiomyofibroblastoma, Fibroepithelial stromal polyps, Myxoid leiomyomas, Myxoid lipomas, Cellular angiofibroma and Smooth muscle tumours should also be taken into consideration in the differential diagnosis for a polypoidal mass arising in the perineum. ${ }^{3}$

Aggressive Angiomyxoma very rarely metastasizes, with a few cases of multiple metastasis ${ }^{4}$ being reported in literature. One case of Vulval Aggressive Angiomyxoma associated with Systemic Lupus Erythematosus has been reported. ${ }^{5}$

Aggressive Angiomyxoma is a myxoid tumour with prominent thick and thin walled vascular component. Spindle and stellate shaped cells maybe seen in a myxoid background with prominent vasculature and no cellular atypia. Immunohistochemical staining is positive for Actin, Desmin, Vimentin, ER, PR and negative for S100 and CEA. ${ }^{6}$

Complete excision of the lesion is done whenever possible, avoiding mutilating surgery, adjunct therapy using arterial embolization and or hormonal treatment maybe required in cases of partial resection of the tumor. Radiotherapy and Chemotherapy are also a part of the 
adjunctive therapy. An alternative treatment modality is the use of Gonadotropin-releasing hormone agonists, which has shown good success in few case reports. ${ }^{7,8}$ Periodic follow-up maybe required upto 15 years after excision for which MRI can be used to detect recurrences. ${ }^{9}$

\section{Conclusion}

Aggressive Angiomyxoma, though a rare entity, should be considered when an insidiously growing painless mass is seen in the vulvovaginal area, especially in premenopausal women in $3^{\text {rd }}$ to $4^{\text {th }}$ decade. High level of suspicion is required in order to make a clinical diagnosis. It is diagnosed mostly based on Histological features. Once optimally treated with surgical excision, less rates of recurrence can be expected. This case of aggressive vulval angiomyxoma is reported due to its rarity of occurrence.

\section{Acknowledgments}

None.

\section{Funding}

None.

\section{Conflicts of interest}

The authors have no conflicts of interest to declare.

\section{References}

1. Steeper TA, Rosai J. Aggressive angiomyxoma of the female pelvis and perineum. Report of nine cases of a distinctive type of gynecologic softtissue neoplasm. Am j surg pathol. 1983;7(5):463-475.
2. Han-Geurts IJ, van Geel AN, van Doorn L, et al. Aggressive angiomyxoma: Multimodality treatments can avoid mutilating surgery. Eur J Surg Oncol. 2006;32:1217-1221.

3. Alameda F, Munné A, Baró T, et al. Vulvar angiomyxoma, aggressive angiomyxoma, and angiomyofibroblastoma: an immunohistochemical and ultrastructural study. Ultrastructural pathology. 2006;30(3):193-205.

4. Siassi RM, Papadopoulos T, Matzel KE. Metastasizing aggressive angiomyxoma. N Engl J Medicine. 1999;341(23):1772.

5. Pahwa P, Khaitan BK, Rao A, et al. Aggressive angiomyxoma of the vulva in a patient with systemic lupus erythematosus. Indian Journal of Dermatology, Venereology, and Leprology. 2012;78(3):361.

6. Barut A, Harma M, Harma M, et al. Giant pedunculated aggressive angiomyxoma of the vulva: report of a case. J Turkish-German Gynecol Assoc. 2009;10:52-54.

7. Calonje E, Fletcher CD. Aneurysmal benign fibrous histiocytoma: clinicopathological analysis of 40 cases of a tumour frequently misdiagnosed as a vascular neoplasm. Histopathology. 1995;26(4):323331.

8. Folpe AL, Fanburgh-Smith JC, Miettinen M , et al. Atypical glomus tumors: analysis of 52 cases, with a proposal for the reclassifi cation of glomus tumors. Am J Surg Pathol 2001;25(1):1-12.

9. Fine BA, Munoz AK, Litz CE, et al. Primary medical management of recurrent aggressive angiomyxoma of the vulva with a gonadotropinreleasing hormone agonist. Gynecol oncology. 2001;81(1):120-122. 